\title{
Multilevel Extended Algorithms in Structural Dynamics on Parallel Computers
}

\author{
Kolja Elssel ${ }^{\mathrm{a}}$ and Heinrich Voss ${ }^{\mathrm{a}}$ \\ aTechnical University of Hamburg - Harburg, \\ Section of Mathematics, D - 21071 Hamburg, Germany,
}

A parallelization concept for the adaptive multi-level substructuring (AMLS) method is presented, the idea of which is to hierarchically substructure the system under consideration, and at the same time to use a truncated eigenvalue decomposition on the interfaces to reduce the excessive number of interface degrees of freedom in component mode methods.

\section{Introduction}

Frequency response analysis of complex structures over broad frequency ranges is very costly, since the stiffness and mass matrices $K$ and $M$ in a finite element model

$K x=\lambda M x$

of a structure under consideration are usually very large. The number of degrees of freedom (DoF) is often reduced to manageable size by condensation and component mode synthesis. However, if the substructuring is chosen fine enough a very large number of interface DoFs appears and the problem remains very large (and much less sparse), whereas for a coarse substructuring a very large number of local modes of the substructures is required to obtain sufficiently accurate approximations to eigenvalues and eigenvectors.

A way out is the adaptive multi-level substructuring where the eigenmodes and eigenfrequencies of the substructures (and their interfaces as well) are approximated by substructuring and modal truncation, and this principle is used in a recursive fashion. Kropp and Heiserer [6] benchmarked an implementation of AMLS against the shift-invert block Lanczos method, and recommend to use AMLS if the dimension of the problem is very large and not only modes corresponding to small eigenfrequencies are needed. They report on a calculation of nearly 2500 eigenmodes of a FE model from vibro-acoustic with 13.500.000 DoF on a HP-RISC workstation. In this paper we present a parallelization concept for the AMLS method.

Our paper is organized as follows. Section 2 briefly sketches condensation with general masters, and Section 3 summarizes the adaptive multi-level substructuring method. In Section 4 we present our parallelization concept using threads and MPI to distribute parts of the problem to computation nodes which operate on separate memory. Section 5 contains a numerical example and discussion.

\section{Parallel condensation with general masters}

We consider the general eigenvalue problem (1) where $K \in \mathbb{R}^{n \times n}$ and $M \in \mathbb{R}^{n \times n}$ are symmetric and positive definite. In the dynamic analysis of structures $K$ and $M$ are the stiffness and the mass matrix of a finite element model of a structure, and they are usually very large and sparse.

To reduce the number of degrees of freedom to manageable size Irons and Guyan independently proposed static condensation, i.e. to choose a small number of degrees of freedom (called masters) which seem to be representative for the dynamic behaviour of the entire structure, and to eliminate the remaining unknowns (called slaves) neglecting inertia terms in some of the equations of (1).

After reordering the equations and unknowns problem (1) can be rewritten as

$\left(\begin{array}{cc}K_{s s} & K_{s m} \\ K_{m s} & K_{m m}\end{array}\right)\left(\begin{array}{c}x_{s} \\ x_{m}\end{array}\right)=\lambda\left(\begin{array}{cc}M_{s s} & M_{s m} \\ M_{m s} & M_{m m}\end{array}\right)\left(\begin{array}{c}x_{s} \\ x_{m}\end{array}\right)$. 
Neglecting the inertia terms in the first equation, solving for the slave unknowns $x_{s}$, and substituting $x_{s}$ into the second equation one obtains the statically condensed problem

$\tilde{K}_{0} x_{m}=\lambda \tilde{M}_{0} x_{m}$

for the master variables $x_{m}$ only, where

$\tilde{K}_{0}:=K_{m m}-K_{m s} K_{s s}^{-1} K_{s m}, \quad$ and

$\tilde{M}_{0}:=M_{m m}-K_{m s} K_{s s}^{-1} M_{s m}-M_{m s} K_{s s}^{-1} K_{s m}+K_{m s} K_{s s}^{-1} M_{s s} K_{s s}^{-1} K_{s m}$.

Combining condensation with substructuring yields a coarse grained parallel algorithm[11] based on the master-worker paradigm. Suppose that the structure under consideration has been decomposed into $r$ substructures, and let the masters be chosen as interface degrees of freedom. Assume that the substructures connect to each other through the master variables only. If the slave variables are numbered appropriately, then the stiffness matrix is given by

$K=\left(\begin{array}{ccccc}K_{s s r} & \ldots & 0 & 0 & K_{s m r} \\ \vdots & \ddots & \vdots & \vdots & \vdots \\ 0 & \ldots & K_{s s 2} & 0 & K_{s m 2} \\ 0 & \ldots & 0 & K_{s s 1} & K_{s m 1} \\ K_{m s r} & \ldots & K_{m s 2} & K_{m s 1} & K_{m m}\end{array}\right)$

and the mass matrix $M$ has the same block form.

It is easily seen that in this case the reduced matrices in (3) are given by

$\tilde{K}_{0}=K_{m m}-\sum_{j=1}^{r} K_{m s j} K_{s s j}^{-1} K_{s m j} \quad$ and

$\tilde{M}_{0}=M_{m m}-\sum_{j=1}^{r}\left(K_{m s j} K_{s s j}^{-1} M_{s m j}+M_{m s j} K_{s s j}^{-1} K_{s m j}-K_{m s j} K_{s s j}^{-1} M_{s s j} K_{s s j}^{-1} K_{s m j}\right)$.

Hence they can be computed completely in parallel, and the only communication that is needed is one fan-in process to determine the reduced matrices $\tilde{K}_{0}$ and $\tilde{M}_{0}$.

This type of condensation has the disadvantage that it produces accurate results only for a small part of the lower end of the spectrum. The approximation properties can be improved substantially if general masters [7] are considered.

Let $z_{1}, \ldots, z_{m} \in \mathbb{R}^{n}$ be independent mastervectors, and let $y_{m+1}, \ldots, y_{n}$ be a complementary basis of $\left\{z_{1}, \ldots, z_{m}\right\}^{\perp}$. With $Z:=\left(z_{1}, \ldots, z_{m}\right)$ and $Y:=\left(y_{m+1}, \ldots, y_{n}\right)$ every $x \in \mathbb{R}^{N}$ can be written as $x=Y x_{s}+Z x_{m}, x_{s} \in \mathbb{R}^{n-m}, x_{m} \in \mathbb{R}^{m}$. Going with this representation into equation (1) and multiplying with the regular matrix $(Y, Z)^{T}$ from the left one obtains the eigenvalue problem

$$
\left(\begin{array}{cc}
Y^{T} K Y & Y^{T} K Z \\
Z^{T} K Y & Z^{T} K Z
\end{array}\right)\left(\begin{array}{c}
x_{s} \\
x_{m}
\end{array}\right)=\lambda\left(\begin{array}{cc}
Y^{T} M Y & Y^{T} M Z \\
Z^{T} M Y & Z^{T} M Z
\end{array}\right)\left(\begin{array}{c}
x_{s} \\
x_{m}
\end{array}\right) .
$$

This decomposition could serve as a basis for condensation with general masters. However, there is a strong practical objection to this naive approach: For large systems the small number of general masters $z_{1}, \ldots, z_{m}$ will be accessible whereas the large number of complementary vectors are definitely not.

Mackens and the second author[7] proved that condensation with general masters can be performed using $Z$ only. Let

$P=K^{-1} Z\left(Z^{T} K^{-1} Z\right)^{-1} Z^{T} Z$.

Then the projected eigenvalue problem

$K_{0} x_{m}:=P^{T} K P x_{m}=\lambda P^{T} M P x_{m}=: \lambda M_{0} x_{m}$, 
is equivalent to the condensed eigenvalue problem with general masters $z_{1}, \ldots, z_{m}$.

If the matrix $Z$ has orthogonal columns, then the projection matrix $P$ can be determined from the augmented linear system

$$
\left(\begin{array}{cc}
K & -Z \\
-Z^{T} & O
\end{array}\right)\left(\begin{array}{c}
P \\
S
\end{array}\right)=\left(\begin{array}{c}
O \\
-I
\end{array}\right)
$$

Similarly as (6) this linear system obtains block form[8] if substructuring is used, if all interface degrees of freedom are chosen as masters, and if they are complemented by general masters the support of each of which is contained in exactly one substructure. Hence, the parallelization concept from [11] applies in this case as well [8].

In particular, choosing a small number of eigenmodes of the clamped substructures (modal masters) additionally to the interface masters (which is equivalent to the component mode method [4]) improves the accuracy of condensation considerably. Pelzer[9] reports for a finite element model of a container ship with ca. 35.000 degrees of freedom the following results. Decomposing the model into 10 substructures und using interface masters only yields a model with 2097 DoF. Approximating the 50 smallest eigenvalues of the structure with this condensed problems yields a maximum relative error of $110 \%$. Adding 170 modal masters of the substructures reduces the maximum relative error to $0.13 \%$. So a small number of additional general masters improves the accuracy considerably. The disadvantage of this parallelization concept however is that we are left with the large number of interface masters to obtain the block structure of system (12). A way out is to reduce the number of interface masters itself using modal informations.

\section{Interface Condensation and Multilevel Extension}

Partitioning the structure FE model into many substructures on multiple levels, obtaining substructure modes up to a specified cutoff frequency, and projecting the problem onto the substructure modal subspace results in the Adaptive Multi-Level Substruture (AMLS) method introduced by Bennighof and co-authors [1], [2], [5].

Using the same reordering as in (6) for the stiffness and mass matrices (K and $\mathrm{M}$ ) and applying a block-Gauss elimination on $K$ yields a block diagonal matrix

$\tilde{K}=\operatorname{diag}\left(K_{s s 1}, \ldots, K_{s s r}, \tilde{K}_{0}\right)$

with $\tilde{K}_{0}$ from definition (7). The same transformation is also applied to the mass matrix M which keeps its structure

$\tilde{M}=\left(\begin{array}{ccccc}M_{s s r} & \ldots & 0 & 0 & \tilde{M}_{s m r} \\ \vdots & \ddots & \vdots & \vdots & \vdots \\ 0 & \ldots & M_{s s 2} & 0 & \tilde{M}_{s m 2} \\ 0 & \ldots & 0 & M_{s s 1} & \tilde{M}_{s m 1} \\ \tilde{M}_{m s r} & \ldots & \tilde{M}_{m s 2} & \tilde{M}_{m s 1} & \tilde{M}_{0}\end{array}\right)$,

but changes all blocks.

$$
\begin{aligned}
\tilde{M}_{0} & =M_{0}+\sum_{i=1}^{r}\left(M_{m s i}\left(-K_{s s i}^{-1} K_{s m i}\right)+\left(-K_{s s i}^{-1} K_{m s i}^{\prime}\right)^{\prime}\left[M_{s m i}+M_{s s i}\left(-K_{s s i}^{-1} K_{s m i}\right)\right]\right) \\
\tilde{M}_{s m j} & =M_{s m j}+M_{s s j}\left(-K_{s s j}^{-1} K_{s m j}\right)
\end{aligned}
$$

Subsequently modal truncation is applied to all substructure pencils $\left(K_{s s j}, M_{s s j}\right)$. By applying the same modal truncation to interface variables $\left(\tilde{K}_{0}, \tilde{M}_{0}\right)$ the dimension of the condensed problem can be reduced considerably.

Without the modal truncation of the interface pencil this algorithm would be similar to the component mode synthesis by Craig and Bampton [4] using fixed interface modes and normal modes. 
The modal truncation for the matrix pencil $\left(\tilde{K}_{0}, \tilde{M}_{0}\right)$ leads to some eigenvectors $\Phi_{0}$ corresponding to the smallest eigenvalues. The vectors

$\Psi_{0}=\left(\begin{array}{c}\tilde{K}_{s m 1} \\ \vdots \\ \tilde{K}_{s m r} \\ I_{n_{0}}\end{array}\right) \Phi_{0}$

are the solutions to the Steklov-Poincaré operator applied to the first eigenmodes of the interface.

\subsection{Multilevel Extension}

The just presented algorithm has in some cases still the drawback of high numbers of interface variables. Choosing the number of substructures as high as the number of processors available might increase the number of interface variables dramatically again. A high imbalance in the blocksizes of all of the $K_{s s i}$ to the $K_{m m}$ block will lead to a high imbalance in computational work (concentrated on the master again) despite the modal truncation on the interface.

This effect can be reduced by extending this algorithm recursively. This can be done for all substructure pencils $\left(K_{s s i}, M_{s s i}\right)$. Some operations in equations $(7,15,16)$ require quantities $\left(M_{s s i}, K_{s s i}\right.$ or $K_{s s i}^{-1}$ ) which might be substructured themselves in the recursive algorithm.

Affected are the terms

$K_{\text {resi }}:=\left(-K_{s s i}^{-1} K_{s m i}\right)$

in equation (7) and

$M_{\text {prodi }}:=M_{s s i}\left(-K_{s s i}^{-1} K_{s m i}\right)=M_{s s i} K_{r e s i}$

in equations $(15,16)$. These terms are implicitly computed in the recursive algorithm. The second term is written as a product with the first term because it only occurs in this form and can safely be computed in advance.

The term " $K_{\text {resi }}$ " can be viewed as solving the (itself again) substructured stiffness matrix $K_{s s i}$ for the right hand side $K_{s m i}$ (under a congruence transformation)

$K_{s s i}=\left(\begin{array}{ccccc}k_{s s r} & \ldots & 0 & 0 & k_{s m r} \\ \vdots & \ddots & \ddots & \vdots & \vdots \\ 0 & \ddots & k_{s s 2} & 0 & k_{s m 2} \\ 0 & \ldots & 0 & k_{s s 1} & k_{s m 1} \\ k_{m s r} & \ldots & k_{m s 2} & k_{m s 1} & k_{m m}\end{array}\right)=\left(\begin{array}{c}K_{s m i_{r}} \\ \vdots \\ \\ K_{s m i_{1}} \\ K_{s m i_{0}}\end{array}\right)$.

This results in simply passing the right hand side to the next lower level of the recursion (appending the columns of $k_{s m i}$ ) for the first $r$ blocks

$K_{r e s i_{j}}=k_{s s j}^{-1} K_{s m i_{j}} j=1, \ldots, r$.

Only the last block is a little more complicated

$K_{r e s i_{0}}=\hat{k}_{m m}^{-1} \hat{K}_{s m i_{0}}:=\left[k_{m m}+\sum_{j=1}^{r} k_{m s j}\left(-k_{s s j}^{-1} k_{s m j}\right)\right]^{-1}\left(K_{s m i_{0}}+\sum_{j=1}^{r} k_{m s j}\left(-k_{s s j}^{-1} K_{s m i_{j}}\right)\right)$.

The local $K_{s m i}$ columns can always be combined with the higher level $K_{s m i}$ columns. Similar considerations apply to the term $M_{\text {prodi }}$.

\section{Parallelization}

The AMLS algorithm has been implemented with the MPI and threads. The MPI is used to distribute parts of the problem to computation nodes which operate on separate memory. Threads are used to employ multiple CPUs on the same shared-memory. 
The program sets up a communication and control loop on each node it runs on for MPI communication and thread scheduling. First on the master and later on all other nodes, the algorithm splits the problem into parts. From each of these parts a new node is created and queued on the local host, or transferred to another host.

The threads can subsequently process each of these nodes. Processing of a node can have the following meanings.

- Determine the number of substructures for this node. This step includes the partitioning if more than one substructure is determined. If only one substructure is determined this part is a leaf of the substructuring tree and results are computed immediately and returned to the parent node.

- Computation of intermediate results. If all results from substructures are present the results of this node are computed and returned to its parent.

Computation of the number of partitions is implemented as a function of dimension, number of nonzero elements in the matrix, recursion level and a predefined minimum (1) leaf) and maximum value for the number of substructures

$r=\left\lfloor 1-2 * \log _{10} \frac{\mathrm{NNZ}}{n^{2}}\right\rfloor$.

This equation leads to 3 substructures for $10 \%$ "fill-in" and 5 substructures for $1 \%$ "fill-in".

The algorithm is implemented recursively passing the following terms between recursion levels

$$
\begin{aligned}
K_{s m} & =\left(K_{s m 1} \ldots K_{s m r}\right)^{T} \\
K_{r e s} & =\left(K_{r e s 1} \ldots K_{r e s r} K_{r e s_{0}}\right)^{T} \\
M_{s m} & =\left(M_{s m 1} \ldots M_{s m r}\right)^{T} \\
M_{\text {mult }} & =M * K_{\text {res }}
\end{aligned}
$$

The recursion is implemented in a depth-first manner. This leads to a small number of tree nodes for which intermediate results have been computed and are in memory at the same time. Memory consumption can be less efficient if multiple threads work on different locations in the tree.

Computation of intermediate results comprises the computation of the local part of the final condensed generalized eigenvalue problem and the return of the results to the parent node.

\section{Results and discussion}

To test the approximation properties of the adaptive multi-level substructuring method we considered the vibrational analysis of a container ship treated already in [10]. Usually in the dynamic analysis of a structure one is interested in the response of the structure at particular points to harmonic excitations of typical forcing frequencies. For instance in the analysis of a ship these excitations are caused by the engine and the propeller, and the locations of interest are in the deckshouse where the perception of the crew is particularly strong.

The finite element model of the ship (a complicated 3 dimensional structure) is not determined by a tool like ANSYS or NASTRAN since this would result in a much too large model. Since in-plane displacements of the ship's surface do not influence the displacements in the deckshouse very much it suffices to discretize the surface by linear membrane shell elements with additional bar elements to correct warping, and to model only the main engine and the propeller as three dimensional structures. For the ship under consideration this yields a very coarse model with 19106 elements and 12273 nodes resulting in a discretization with 35262 degrees of freedom.

We consider the structural deformation caused by an harmonic excitation at a frequency of $4 \mathrm{~Hz}$ which is a typical forcing frequency stemming from the engine and the propeller. Since the deformation is small the assumptions of the linear theory apply, and the structural response can be determined by the mode superposition method taking into account eigenfrequencies in the range between 0 and $7.5 \mathrm{~Hz}$ (which corresponds to the 50 smallest eigenvalues for the ship under consideration). Since the finite element model is very coarse the accuracy requirements are very modest, and an error of $10 \%$ for the natural frequencies often suffices [3]. 
To determine these eigenvalues with the required accuracy the AMLS method generated a substructuring with 5 levels where the cutoff frequency for eigenmodes of the substructures and the interfaces was chosen to be 8250 . The dimension of the reduced eigenvalue problem was 172 , where $19,64,61$, 28 and 0 eigenmodes of substructures or interface patches of the levels 0 to 4 , respectively, contributed to the reduced model. Generating and solving this problem on an Intel XEON machine with two 2.2 $\mathrm{GHz}$ processors and $5 \mathrm{~GB}$ shared memory required a CPU time of 150 seconds using one processor and 81 seconds with both processors yielding a speedup of 1.85 .

For comparison, the same eigenvalue problem was considered in [10] using condensation with general masters. To meet the accuracy requirements there a partition into 10 natural substructures had to be augmented by 50 general masters obtained by reanalysis techniques (yielding a condensed problem with 2147 DoF). Hence, AMLS needs a much smaller reduced model than condensation with general masters and classical substructuring, and it is worth to be studied further.

Our future research will concentrate on two issues: First, a uniform cutoff frequency is used for all substructures and all interface patches. Can the approximation quality be improved by some strategy to choose cutoff frequencies individually (for instance, depending on the size or on the level)? Second, the partitioning is generated depending only on the graph of the stiffness matrix. Is it possible to improve the accuracy (and hence reduce the dimension of the reduced model for a given accuracy requirement) taking into account geometric properties of the domain (reentrant corners) or the behaviour of (already approximated) eigenmodes ?

\section{Acknowledgements}

Thanks are due to Christian Cabos, Germanischer Lloyd, who provided us with the finite element model of the container ship. The first author gratefully acknowledges financial support of this project by the German Foundation of Research (DFG) within the Graduiertenkolleg "Meerestechnische Konstruktionen".

\section{REFERENCES}

[1] J.K. Bennighof, M.F. Kaplan, M.B. Muller, M. Kim, Meeting the NVH computational challenge: Automated Multi-Level Substructuring. Proceedings of the $18^{\text {th }}$ International Modal Analysis Conference, San Antonio, Texas, 2000

[2] J.K. Bennighof, R. Lehoucq, An automated multilevel substructuring method for eigenspace computation in linear elastodynamics. Tech.Rep. SAND2001-3279J. Sandia National Laboratory 2001

[3] C. Cabos, Private communication 2001

[4] R.R. Craigh, Jr., and M.C.C. Bampton, Coupling of substructures for dynamic analysis. AIAA Journal 3, 1313 - 1319 (1968)

[5] M.F. Kaplan, Implementation of Automated Multilevel Substructuring for Frequency Response Analysis of Structures. Ph.D. thesis, University of Texas at Austin 2001

[6] A. Kropp and D. Heiserer, Efficient broadband vibro-acoustic analysis of passenger car bodies using an FE-based component mode synthesis approach. In H.A. Mang, F.G. Rammerstorfer, J. Eberhardsteiner (eds.), Proceedings of Fifth World Congress on Computational Mechanics, Vienna, Austria 2002

[7] W. Mackens and H. Voss, Nonnodal condensation of eigenvalue problems, ZAMM 79, $243-255$ (1999)

[8] W. Mackens and H. Voss, General masters in parallel condensation of eigenvalue problems, Parallel Computing 25, 893 - 903 (1999)

[9] A.M. Pelzer, Systematische Auswahl von Masterfreiheitsgraden für die parallele Kondensation von Eigenwertaufgaben. Shaker Verlag, Aachen 2001

[10] A. Pelzer and H. Voss, A Parallel Condensation-Based Method for the Structural Dynamic Reanalysis Problem. pp. 346 - 353 in G.R. Joubert, A. Murli, F.J. Peters, M. Vanneschi (eds.), Parallel Computing: Advances and Current Issues, Proceedings of ParCo2001, Imperial College Press, 2002

[11] K. Rothe and H. Voss, A fully parallel condensation method for generalized eigenvalue problems on distributed memory computers. Parallel Computing 21, 907 - 921 (1995) 